\title{
A novel approach for electrical circuit modeling of Li-ion battery for predicting the steady-state and dynamic I-V characteristics
}

\author{
SAURABH SAXENA*, S RAGHU RAMAN, B SARITHA and VINOD JOHN \\ Department of Electrical Engineering, Indian Institute of Science, Bangalore 560012, India \\ e-mail: saurabh.2857.saxena@gmail.com
}

MS received 5 November 2014; revised 25 December 2015; accepted 30 December 2015

\begin{abstract}
A novel approach for electrical circuit modeling of Li-ion battery is proposed in this paper. The model proposed in this paper is simple, fast, not memory intensive and does not involve any look-up table. The model mimics the steady-state and dynamic behavior of battery. Internal charge distribution of the battery is modeled using two RC circuits. Self-discharge characteristic of the battery is modeled using a leakage resistance. Experimental procedure to determine the internal resistance, leakage resistance and the value of RC elements is explained in detail. The variation of parameters with state of charge (SOC) and magnitude of current is presented. The internal voltage source of the battery model varies dynamically with SOC to replicate the experimental terminal voltage characteristics of battery. The accuracy of model is validated with experimental results.
\end{abstract}

Keywords. Battery modeling; internal impedance; lithium-ion battery; open-circuit voltage; self-discharge; state of charge.

\section{Introduction}

Electric vehicles (EVs), plug-in hybrid electric vehicles (PHEVs), renewable energy systems, portable consumer electronic gadgets like smart phones and laptops, vehicle to grid (V2G) and grid to vehicle (G2V) technologies heavily depend on energy storage devices. Batteries are preferred for energy storage applications. Over the past decade, Lithium ion (Li-ion) has emerged as the most prominent rechargeable battery chemistry [1-3]. High specific energy, longevity, low toxicity and maintenance, minimum selfdischarge and absence of memory effect are the features which make Li-ion battery chemistry as the energy storage element of choice. Li-ion batteries have very low tolerance for over-charge/discharge and hence there is a mandatory requirement for an embedded protection circuit to keep voltage and current within permissible limits [4-7]. All batteries including $\mathrm{Li}$-ion are subjected to aging. Batteries experience permanent reduction in usable capacity over a period of time due to irreversible physical and chemical changes that are associated with usage [8-11]. Overall, Liion batteries have features that ideally befit EVs and PHEVs.

Modeling and simulation form an integral part for analyzing and prototyping EVs/PHEVs. Battery models are a vital component of a dynamic electric vehicle simulator [12]. Battery modeling plays an influential role in design

*For correspondence and in the estimation of battery performance. System engineers need to integrate batteries to a larger system and evaluate its performance through simulations. Battery pack designers need to optimize the physical structure of the pack to maximize the performance. Control engineers need to develop algorithms for Battery Management Systems (BMS) in which batteries are an indispensable part of a complex system that provides vital information to other controllers. It is therefore necessary to develop battery models which precisely mimic real battery characteristics. The models must be fast, highly accurate, simple, less memory intensive and must mimic the characteristics that define a battery. Battery models that are included in EVs/ HEVs must be capable of accurately predicting the SOC and $\mathrm{SOH}$ to improve the reliability of the vehicle.

Battery modeling is a multi-disciplinary research area which involves chemistry, material science, physics and engineering. This has led to several modeling approaches: electrochemical, mathematical and electrical. Electrochemical models [13-15] describe the battery chemistry in great detail making these models highly accurate. However, these models tend to become too complex as a set of partial differential equations describing the ion transport and diffusion phenomena, mass and charge balance, ion distribution and temperature effects have to be solved to find the behavior of battery. Reduction in the computational complexities of these models while maintaining the accuracy of state estimation is still an active area of research. These models also require large number of parameters based on 
the knowledge of the structure, chemical composition, capacity, temperature, and other characteristics [16] that are difficult to obtain.

Mathematical models adopt empirical equations or math based stochastic models [17-19]. The model presented by Chiasserini and Rao [17] takes into account of only the charge recovery effect and neglects all other characteristics. It is able to represent pulse discharge only. The models suggested by Rakhmatov et al [18] and Rong and Pedram [19] are accurate in predicting battery lifetime and remaining capacity of battery respectively. Both models rely on the high-level representation of battery with analytical expressions based on physical laws. These models are computationally complex and suitable for predicting only individual behavioral aspects of the battery.

Electrical or circuit models can be further classified as ac impedance-based [20, 21], run-time based [22, 23] and Thevenin-based [24-27]. In ac impedance-based model, the parameters are determined using impedance spectroscopy method. These models use complex impedance network to match the battery ac response. The conversion of these models from frequency-domain to time-domain is difficult as all the complex impedance elements cannot be easily approximated by RC elements. Hence the ability of these models to match the complex transient characteristics of the battery is also limited. These models also work only at fixed SOC value. Run-time based models use coupled electrical networks and look-up tables to determine SOC, battery runtime, cell voltage and thermal characteristics. However, these models are substantially complex and inaccurate in predicting battery characteristics. In Thevenin-based models, parameters can be obtained by pulse discharge tests. They can mimic the transient and steady-state behavior of the battery. SOC can be determined using coulomb counting procedure [26, 27] or extended Kalman filter [24]. Modeling the steady-state and transient behavior of battery using Thevenin-based circuit model is the focus of this paper.

Various forms of Thevenin-based battery model are shown in figure 1 . These models involve the controllable voltage source (CVS) and passive $\mathrm{R}, \mathrm{C}$ components to model the battery. The model in figure $1 \mathrm{a}$ is not capable of mimicking the transient characteristics of the battery [24, 27]. The three models given in figure 1 are compared by Einhorn et al [26] with two different parameterization approaches: linear, look-up table and it is concluded that linear approximation is computationally efficient. In general, the electrical models presented by Chen et al [24], Coleman et al [25], Einhorn et al [26], and Chen and Rincon-Mora [28] do not consider the difference between CVS and OCV, which is prominent at higher current rates.

Zhang and Chow [29] proved that the model in figure 1c was computationally efficient and accurate for modeling the dynamic characteristics of the battery. Hence, in this paper model in figure $1 \mathrm{c}$ is considered for modeling the practical $\mathrm{I}-\mathrm{V}$ characteristics of the battery. This model embraces the

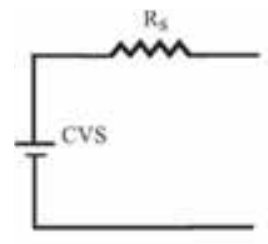

(a)

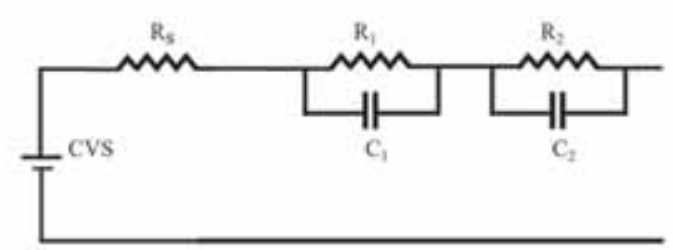

(c)

(b)

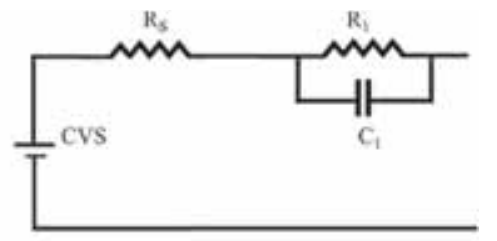

Figure 1. Electrical circuit model with (a) one resistance, (b) one resistance and one RC network, and (c) one resistance and two RC networks.

features of both analytical and electrical modeling approaches. The model is simple and not memory intensive as it does not use a look-up table. The proposed model is capable of mimicking the relaxation phenomenon of battery for a wide time period of $300 \mathrm{~s}$. The steady-state and transient $\mathrm{I}-\mathrm{V}$ performance of battery is convincingly replicated with the model. The internal parameters are experimentally determined and their variation with SOC and current is reported. For computational efficiency, the parameters are considered constant in the model for the practical range of SOC from $30 \%$ to $100 \%$. Battery internal voltage represented as CVS is experimentally shown to be different from OCV and it is also modeled as a function of SOC. Experimentally measured self-discharge effect has been included in the model. Battery terminal voltage is represented as BTV. A normalized approach for considering the battery parameters is adopted in the paper. This helps to ensure that the simplification of the battery model does not compromise on its accuracy.

The paper is organized as follows: Thevenin-based circuit model has been described in section 2. Section 3 elaborates on the experiments carried out on Li-ion batteries. This includes determination of OCV versus SOC, BTV versus SOC and extraction of battery parameters by pulse-discharge and self-discharge tests. Section 4 provides the validation of proposed model with experimental data.

\section{Thevenin-based circuit model}

Electrical circuit models are most suited for electrical engineering simulation purposes. These models use a combination of voltage sources, resistors, capacitors and inductors. Circuit models can directly be connected to another electrical system for simulation. figure 1 shows different Thevenin-based circuit models, which are available in literature [24-28]. 
In figure $1, R_{\mathrm{S}}$ is the net resistance of the electrolyte, two electrodes and contacts. $R_{1}, R_{2}, C_{1}$ and $C_{2}$ are used to characterize the transient behavior of the battery. The internal charge distribution within the battery is represented by two RC networks having the time constants $\left(\tau_{1}=R_{1}{ }^{*} C_{1}\right.$ and $\tau_{2}=R_{2} * C_{2}$ ) in the order of seconds and minutes respectively. CVS is the internal voltage source of the battery, which is modeled as OCV characteristics of the battery in the literature [24-26, 28]. In this paper, CVS is experimentally shown to differ from OCV, depending on the magnitude of current. Here CVS is modeled as a second-order polynomial function of SOC, which mimics the terminal characteristics of battery under all static and dynamic conditions.

\section{Battery characteristics}

The voltage characteristics of the battery are OCV versus SOC and BTV versus SOC for various discharge currents. The impedance characteristics of the battery include series resistance and $\mathrm{RC}$ network impedance. The experimental determination of voltage and impedance characteristics of the battery is explained in the following subsections.

\subsection{Normalized battery parameters}

Experiments are carried out on 3.7 V, $2200 \mathrm{mAh} \mathrm{Li-ion}$ batteries. A per-unit (pu) system of representation of battery parameters has been adopted in the paper as it helps in visualizing the errors due to variation in a particular parameter. The base resistance $\left(R_{\mathrm{B}}\right)$ is calculated as the ratio of nominal voltage of battery to $1 \mathrm{C}$ current, which is equal to $1.68 \Omega$. The base capacitance or equivalent capacitance $\left(C_{\mathrm{B}}\right)$ of this Li-ion battery is $2140 \mathrm{~F}$. Battery current is generally specified in C-rates. C-rate signifies a charge or discharge rate equal to the battery capacity divided by one hour. For example, $1 \mathrm{C}$ for a $2200 \mathrm{mAh}$ battery would be $2.2 \mathrm{~A}, \mathrm{C} / 5$ for the same battery would be $440 \mathrm{~mA}$ and $2 \mathrm{C}$ would be $4.4 \mathrm{~A}$.

\subsection{Open-circuit voltage versus state of charge}

The settled voltage across the battery terminals under no load condition is known as OCV or steady-state open-circuit terminal voltage. OCV varies in direct proportion with SOC of the battery.

A fully charged (100\% SOC) Li-ion battery is discharged through an electronic load for a defined time period ' $t_{\mathrm{dis}}$ '. After the discharge time $t_{\text {dis }}$, the battery is allowed to settle down for an hour. The constant voltage that appears across the battery terminals after the settling period is the OCV. Batteries must not be discharged below the cut-off voltage $\left(V_{\mathrm{C}}\right)$. The value of $V_{\mathrm{C}}$ is specified as $3 \mathrm{~V}$ in the datasheet of the battery.

During experiment, the battery is connected to $1.5 \mathrm{~A}$ current sink for a discharge period of $5 \mathrm{~min}$. To obtain better resolution, the discharge period is reduced to $1 \mathrm{~min}$ in the non-linear SOC regions of 0-10 and 90-100\%. When fully charged, Li-ion battery has an OCV of $4.2 \mathrm{~V}$ and it corresponds to $100 \%$ SOC.

In this paper, the coulomb counting approach is used for SOC determination. The capacity or charge $\left(Q_{\text {dis } 1}\right)$ removed from the battery, due to the flow of constant current $I_{1}$, for time $t_{\mathrm{dis} 1}$ is given below:

$$
Q_{\mathrm{dis} 1}=\int_{0}^{t_{\mathrm{dis} 1}} i \mathrm{~d} t=I_{1} * t_{\mathrm{dis} 1} .
$$

The value of $\mathrm{SOC}_{1}$ of the battery after the removal of charge $Q_{\text {dis1 }}$ is given by

$$
\mathrm{SOC}_{1} \%=100 *\left(1-\frac{Q_{\mathrm{dis} 1}}{Q_{N}}\right)
$$

where $Q_{\mathrm{N}}$ is the nominal capacity of the battery and can be found from the datasheet. $Q_{\mathrm{N}}$ can also be experimentally determined by continuous discharge tests. The total charge removed from the battery after two successive discharges is given by

$$
Q_{\mathrm{dis} 2}=Q_{\mathrm{dis} 1}+I_{2} * t_{\mathrm{dis} 2} .
$$

Similarly, the reduction in the capacity of the battery after ' $n$ ' discharge iterations and the corresponding SOC of the battery is given by following equations:

$$
\begin{gathered}
Q_{\operatorname{dis}(n)}=Q_{\operatorname{dis}(n-1)}+I_{n} * t_{\text {disn }} \\
\operatorname{SOC}_{(n)} \%=100 *\left(1-\frac{Q_{\operatorname{dis}(n)}}{Q_{N}}\right) .
\end{gathered}
$$

After every discharge, the OCV and SOC of the battery are determined. figure 2 shows the variation of OCV with SOC of the battery.

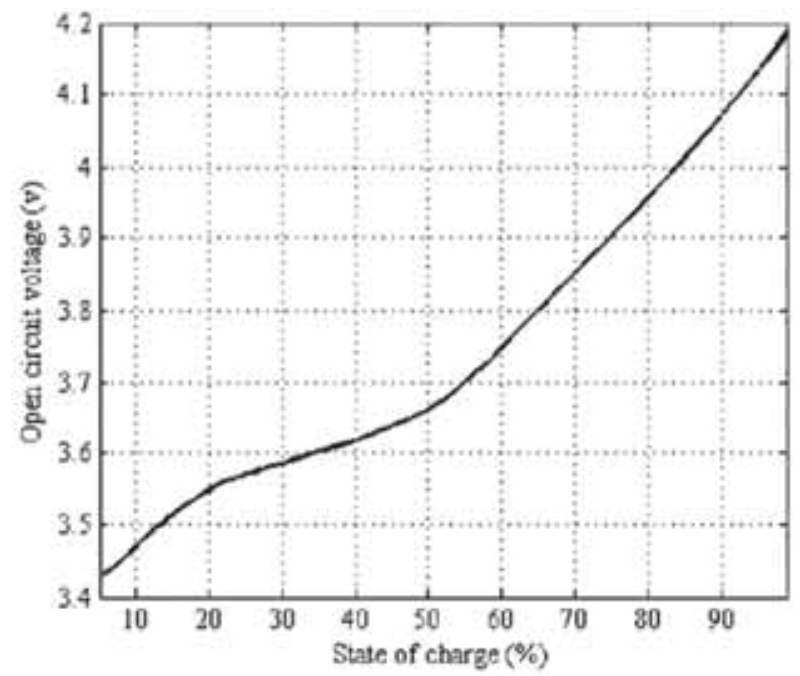

Figure 2. Measured OCV versus SOC characteristics for Li-ion cell. 


\subsection{Battery terminal voltage versus state of charge}

Battery terminal voltage (BTV) is the voltage across the leads of the battery while serving the load. Determination of BTV versus SOC is relatively easier, as shown in figure 3. The electronic load is programmed to behave as a constant current sink and connected across the terminals of the battery till the battery discharges to its cut-off voltage. Battery terminal voltage and the discharge current are continuously recorded in a data logger. SOC is calculated in accordance with Eqs. (1-5).

The experiment is conducted over a wide range of loads, to investigate the difference in discharge pattern. The range is chosen such that the maximum discharge current does not exceed the prescribed value in the datasheet. Figure 4 shows BTV versus SOC plot for various C-rates of the battery.

\subsection{Procedure to determine internal parameters}

Internal impedance parameters can be determined using impedance spectroscopy [21] and also by pulse discharge technique [28]. Pulse discharge technique is used in this paper as it is simple and the procedure is

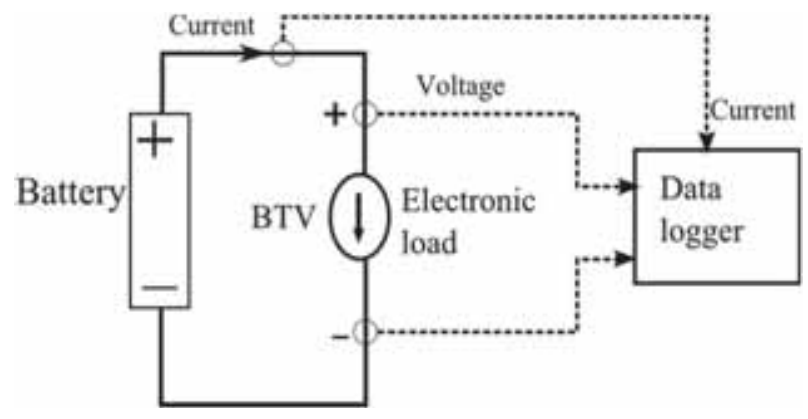

Figure 3. Experimental set-up for BTV measurements.

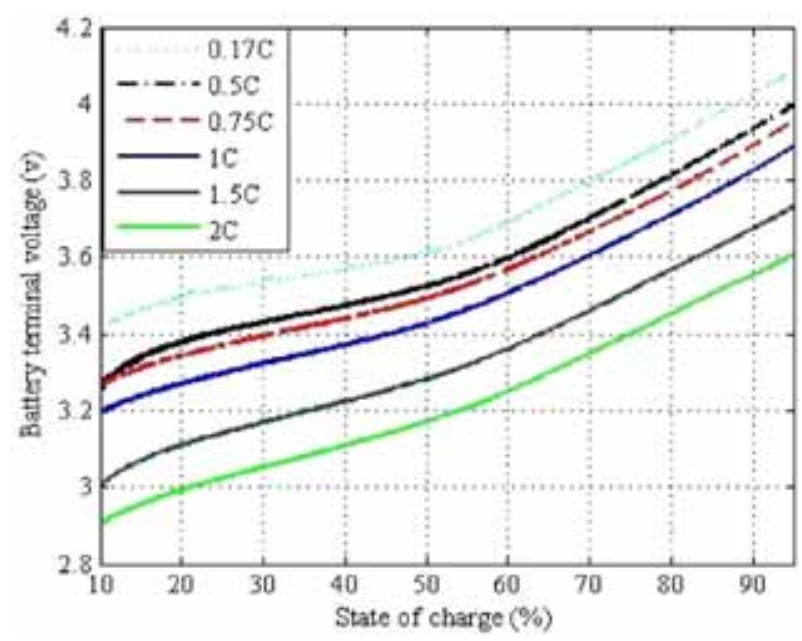

Figure 4. Measured BTV versus SOC curve of a Li-ion cell. discussed herein. The battery is fully charged and a known constant current load is applied across it. The load is alternately connected and disconnected using an electronic switch. BTV and the discharge current are continuously recorded in a data logger. The experiment is repeated for various $\mathrm{C}$-rates at room temperature. The value of internal series resistance $R_{\mathrm{S}}$ (figure 1c) is calculated by sudden drop in terminal voltage of battery due to the pulse current. The change in BTV with pulse discharge current is shown in figure 5.

With reference to figure 5 , the value of $R_{\mathrm{S}}$ is given by

$$
R_{\mathrm{s}}=\frac{V_{\mathrm{a}}-V_{\mathrm{b}}}{i} \Omega
$$

where $V_{\mathrm{a}}$ is the BTV just before the beginning of discharge, $V_{\mathrm{b}}$ is the BTV just after the beginning of discharge, and $i$ is the instantaneous rise in discharge current.

The variation of $R_{\mathrm{S}}(\mathrm{pu})$ with SOC is shown in figure 6 . From figure 6 , it can be inferred that $R_{\mathrm{S}}$ is almost constant from $40 \%$ to $100 \%$. At low value of SOC, the resistance increases and hence the efficiency of battery drops at low value of SOC. The variation of $R_{\mathrm{S}}$ with discharge current is not significant and hence the value of $R_{\mathrm{S}}$ in the simulation model can be taken as constant.

In figure 5, the load is disconnected at $240 \mathrm{~s}$ and the sudden increase in voltage from $V_{\mathrm{s}}$ to $V_{\mathrm{r}}$ can be observed. The sudden change in voltage is due to drop in the internal series resistance. It can also be observed from figure 5 that the terminal voltage of the battery changes from $V_{\mathrm{r}}$, without any load across it. This phenomenon is due to internal charge distribution within the battery and it is termed as relaxation phenomenon. The internal charge distribution is modeled using two RC networks and the value of $R_{1}, C_{1}, R_{2}$ and $C_{2}$ are determined from the relaxation voltage waveform (figure 5). During relaxation period, the charge stored across $C_{1}\left(C_{2}\right)$ is dissipated to $R_{1}\left(R_{2}\right)$. The voltage across parallel $R_{1} C_{1}\left(R_{2} C_{2}\right)$ circuit is modeled using exponential waveform. From the least-square curve-fit of experimental

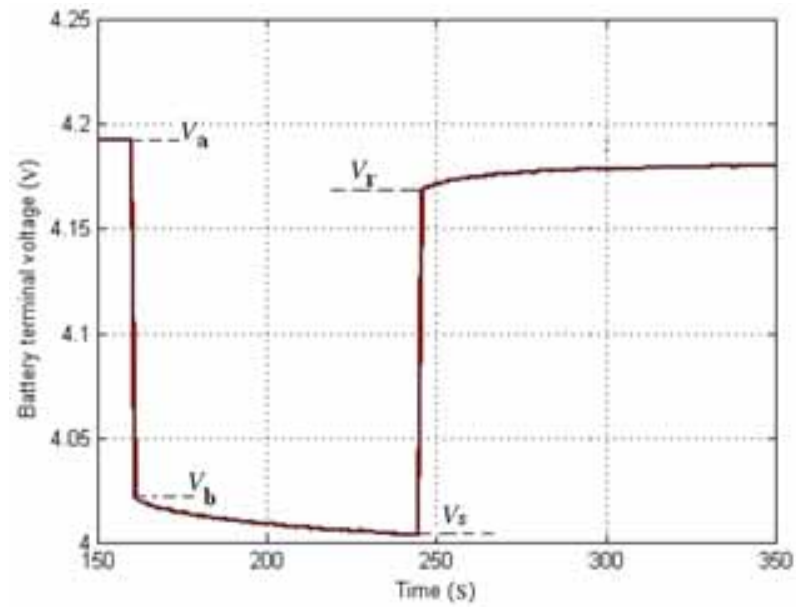

Figure 5. Change in terminal voltage of battery under pulse current. 


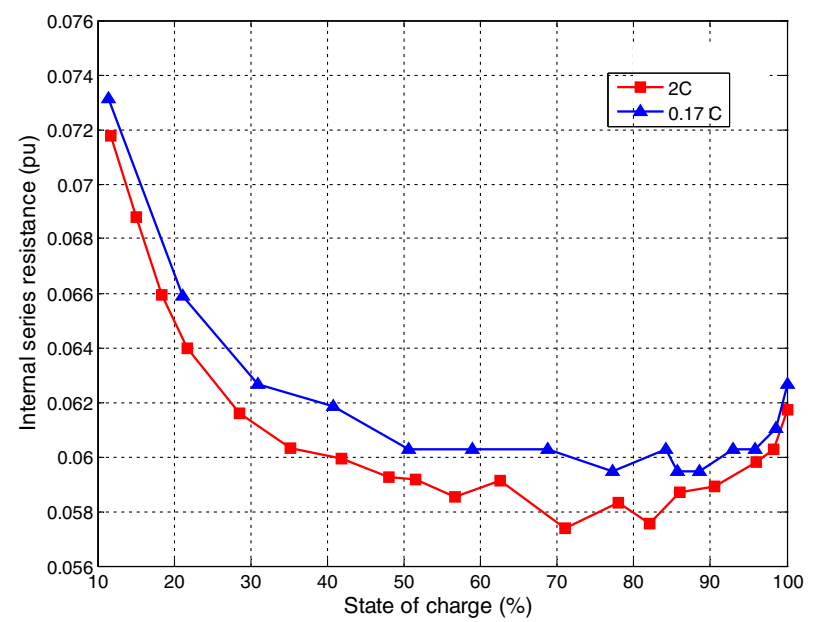

Figure 6. Variation of series resistance of battery with state of charge.

data, the value of internal impedance $\left(R_{1}, C_{1}, R_{2}\right.$ and $\left.C_{2}\right)$ is calculated [30]. The variation of internal impedance (pu) of the battery with SOC and discharge current is shown in figure 7 .

From figure 7 it can be observed that the variation of parameters $R_{1}, R_{2}, C_{1}$ and $C_{2}$ over full SOC range for different C-rates has both the increasing and decreasing trends. To maintain the simplicity of model, mean of all the values of $R_{1}, R_{2}, C_{1}$ and $C_{2}$ corresponding to different $\mathrm{C}$-rates has been taken. These mean values (per-unit) are given in table 1. From table 1, it can be seen that mean values of impedance parameters for different C-rates are quite close. Maximum error is $0.002 \mathrm{pu}$ for resistance and $0.54 \mathrm{pu}$ for capacitance. Hence an overall mean considering all the discharge rates can be taken for different impedance parameters. These mean values have been used in the proposed simulation model.

\subsection{Self-discharge of Li-ion batteries}

All battery chemistries including Li-ion exhibit self-discharge (SD) phenomenon. Loss of battery capacity when the battery is in an open-circuit condition is called SD. The variation of open-circuit voltage of Li-ion battery with time is shown in figure 8. It is observed that the decay in voltage is exponential. The SD pattern among commercially available $\mathrm{Li}$-ion batteries is non-uniform. This is due to difference in materials used to manufacture the battery, designs and protection circuits.

The effect of SD can be included into the simulation model by including a parallel resistance $\left(R_{\mathrm{SD}}\right)$ across the controllable voltage source. The controllable voltage source can be considered as an equivalent capacitor $\left(C_{\mathrm{B}}\right)$. From the capacity and nominal voltage, the equivalent capacitance of the battery can be determined. The equivalent capacitance $\left(C_{\mathrm{B}}\right)$ is $2140 \mathrm{~F}$. The decay in open-circuit voltage is modeled using the following equation:
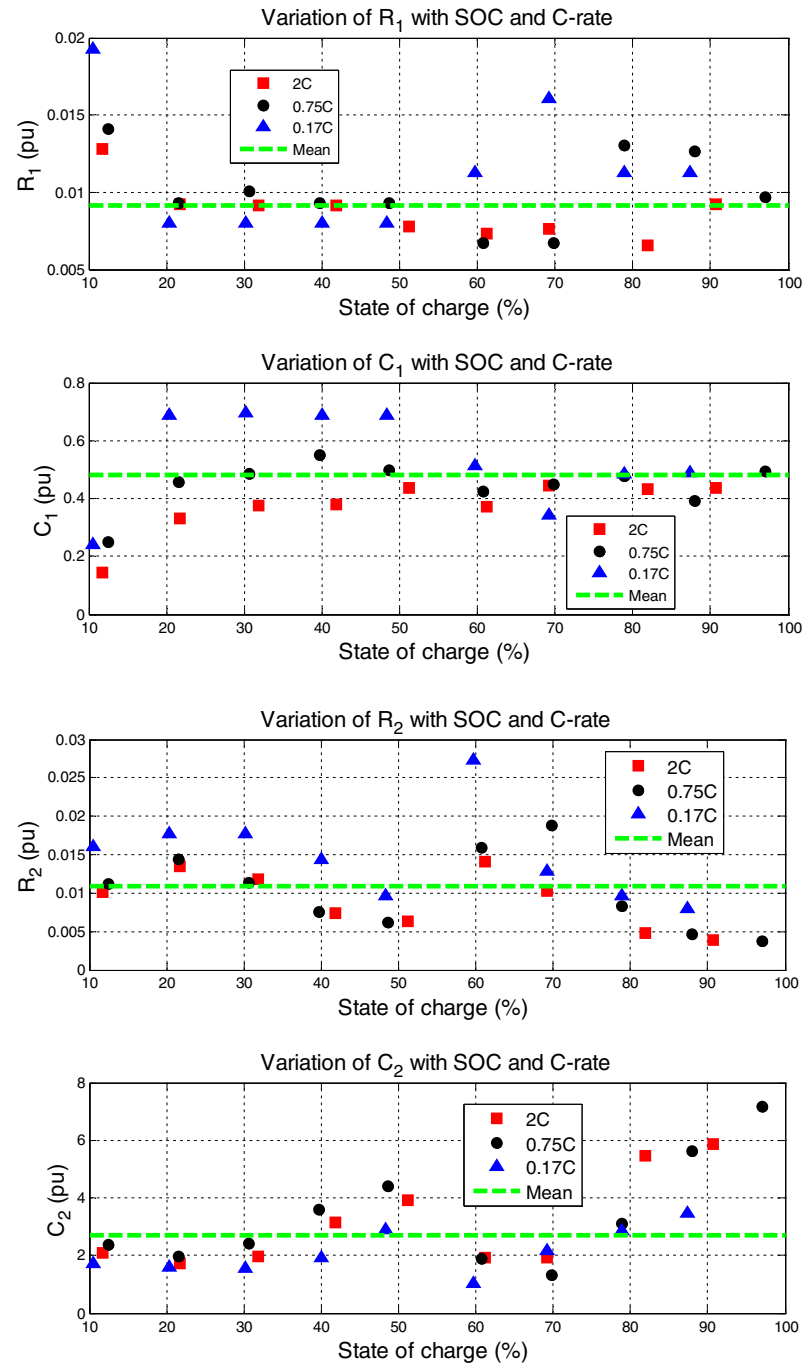

Figure 7. Variation of internal impedance of battery with SOC and C-rate.

Table 1. Mean values of $R_{1}, R_{2}, C_{1}, C_{2}$ in per-unit for different C-rates.

\begin{tabular}{lcccc}
\hline & \multicolumn{4}{c}{ Mean } \\
\cline { 2 - 5 } C-rate & $R_{1}(\mathrm{pu})$ & $C_{1}(\mathrm{pu})$ & $R_{2}(\mathrm{pu})$ & $C_{2}(\mathrm{pu})$ \\
\hline 0.17 & 0.0094 & 0.57 & 0.0129 & 2.19 \\
0.75 & 0.0096 & 0.47 & 0.0109 & 3.04 \\
2 & 0.0083 & 0.4 & 0.009 & 3.23 \\
All discharge rates & 0.0091 & 0.48 & 0.0109 & 2.69 \\
\hline
\end{tabular}

$$
V_{0}=V_{1} * e \frac{t}{\tau}
$$

where $V_{0}$ is the initial voltage, $V_{1}$ is the voltage after 60 days and $\tau=R_{\mathrm{SD}} * C_{\mathrm{B}}$

From Eq. (7), the value of $\tau$ and hence $R_{\mathrm{SD}}$ is determined i.e. $R_{\mathrm{SD}}=0.35 \mathrm{M} \Omega$. 


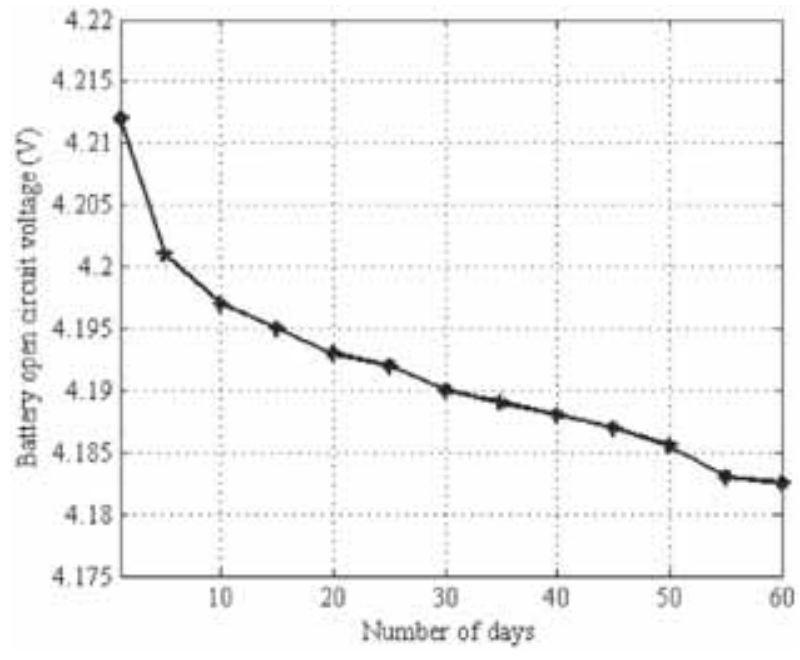

Figure 8. Measured SD for a Li-ion battery.

\subsection{Controllable voltage source}

Magnitude of controllable voltage source CVS is equal to the sum of voltage drop in internal impedance of the battery and BTV. BTV is the measured voltage, with the load connected across the battery. The determination of internal parameters is discussed in Section 3.4. From the measured discharge current $i$, terminal voltage BTV and impedance parameters; the value of CVS can be calculated as

$$
\mathrm{CVS}=\mathrm{BTV}+V_{\mathrm{r}}+V_{\mathrm{c} 1}+V_{\mathrm{c} 2} .
$$

As discussed in section 3.4, the internal impedance parameters vary with discharge current and SOC. To make the model simple, the variation of internal impedance with discharge rate and SOC is not considered; $R_{\mathrm{S}}=101.4 \mathrm{~m} \Omega$, $R_{1}=15.4 \mathrm{~m} \Omega, \quad R_{2}=18.3 \mathrm{~m} \Omega, \quad C_{1}=1028.7 \mathrm{~F}, \quad C_{2}=$ $5756.6 \mathrm{~F} . V_{\mathrm{C} 1}$ and $V_{\mathrm{C} 2}$ represent the voltage across impedance elements (figure 10). Also the cyclic efficiency is assumed to be equal to 1 . The variation of CVS with SOC is plotted in figure 9. From figure 9, it is clear that CVS is close to OCV for lower C-rate and it varies considerably for higher C-rate current. Table 2 shows the quantitative difference between CVS and OCV at different SOC and C-rates. $E_{\mathrm{v}}$ denotes the error between calculated CVS and OCV. $E_{\mathrm{z}}$ is the error due to the assumption of constant values of internal impedance parameters. From table 2, it is clear that $E_{\mathrm{v}}$ is always higher than $E_{\mathrm{z}}$. It signifies that the difference between CVS and OCV is not due to the constant internal impedance parameters. Hence, CVS in the proposed simulation model (figure 10) is taken different from $\mathrm{OCV}$, to reproduce the experimental terminal characteristics of the battery.

To make the model simple, CVS over various C-rates is averaged and the variation of CVS with SOC is expressed as a second degree polynomial function:

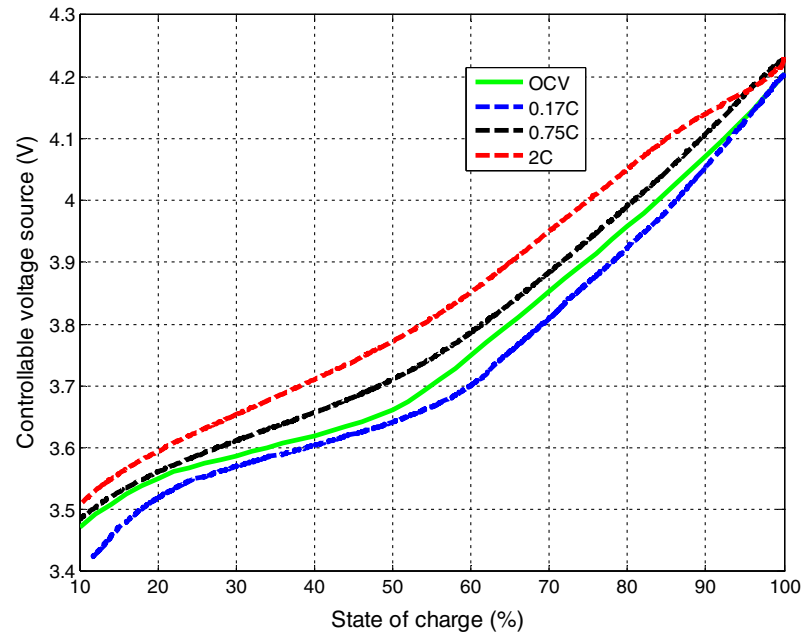

Figure 9. Variation of CVS with SOC and C-rate.

Table 2. Difference between OCV and CVS for various C-rates.

\begin{tabular}{lcccrr}
\hline C-rate & SOC $(\%)$ & OCV $(\mathrm{V})$ & CVS $(\mathrm{V})$ & $E_{\mathrm{v}}(\%)$ & $E_{\mathrm{z}}(\%)$ \\
\hline 0.17 & 69.15 & 3.844 & 3.8 & 1.19 & 0.15 \\
& 48.41 & 3.654 & 3.635 & 0.51 & -0.04 \\
& 20.37 & 3.552 & 3.522 & 0.81 & 0.19 \\
0.75 & 69.84 & 3.851 & 3.883 & -0.86 & 0.50 \\
& 48.68 & 3.656 & 3.703 & -1.27 & -0.12 \\
& 21.57 & 3.559 & 3.57 & -0.30 & 0.81 \\
2 & 69.21 & 3.844 & 3.943 & -2.68 & -0.98 \\
& 51.17 & 3.67 & 3.78 & -2.97 & -1.39 \\
& 21.79 & 3.56 & 3.606 & -1.24 & 1.28 \\
\hline
\end{tabular}

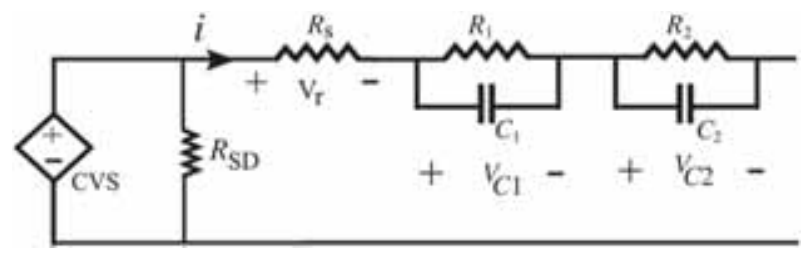

Figure 10. Proposed electrical circuit model for the battery.

$$
\mathrm{CVS}=5.56 \mathrm{e}-05 * \mathrm{SOC}^{2}+0.001788 * \mathrm{SOC}+3.491
$$

Table 3 considers the averaging of CVS over various C-rates. During the experiments, C-rates of $0.17 \mathrm{C}, 0.5 \mathrm{C}$, $0.75 \mathrm{C}, 1 \mathrm{C}, 1.5 \mathrm{C}$, and $2 \mathrm{C}$ have been considered. The values of CVS in practical range of SOC are found to be increasing with increase in C-rate (figure 9). Hence, the error between the modeled CVS and actual CVS corresponding to minimum and maximum C-rates is calculated to check the accuracy of averaging method. $E_{1}$ and $E_{\mathrm{h}}$ denote the low and high error between modeled CVS and 
Table 3. Error between actual and modeled CVS values.

\begin{tabular}{llllll}
\hline & \multicolumn{5}{c}{ CVS (V) } \\
\cline { 2 - 4 } SOC & Model & $0.17 \mathrm{C}$ & $2 \mathrm{C}$ & $E_{\mathrm{l}}(\%)$ & $E_{\mathrm{h}}(\%)$ \\
\hline 90 & 4.102 & 4.055 & 4.141 & -1.27 & 1.05 \\
80 & 3.990 & 3.924 & 4.051 & -1.78 & 1.65 \\
70 & 3.889 & 3.81 & 3.951 & -2.14 & 1.68 \\
60 & 3.798 & 3.701 & 3.852 & -2.62 & 1.46 \\
50 & 3.719 & 3.641 & 3.773 & -2.11 & 1.46 \\
40 & 3.651 & 3.604 & 3.711 & -1.27 & 1.62 \\
30 & 3.595 & 3.570 & 3.654 & -0.68 & 1.59 \\
20 & 3.549 & 3.519 & 3.594 & -0.81 & 1.22 \\
\hline
\end{tabular}

CVS values calculated from measurements for $0.17 \mathrm{C}$ and $2 \mathrm{C}$ rates respectively. From table 3 , it can be observed that the maximum error is limited to $2.62 \%$. The averaging of CVS values also ensures that the model works for both high as well as low C-rates. This will not be the case if CVS has been modeled as OCV which is close to CVS values only at low C-rates.

\section{Validation of the model}

The proposed battery model with CVS and internal impedance is shown in figure 10. The internal series resistance is almost constant over useful range of SOC. The value of series resistance $R_{\mathrm{S}}$ is higher at the low range of SOC $(0-30 \%)$; hence, the efficiency of the battery is poor. The useful operating range of SOC of the battery is from $30 \%$ to $100 \%$ and hence it is considered for comparing and validating the simulation model. RC elements are averaged over various $\mathrm{C}$-rates and useful range of SOC. The model is

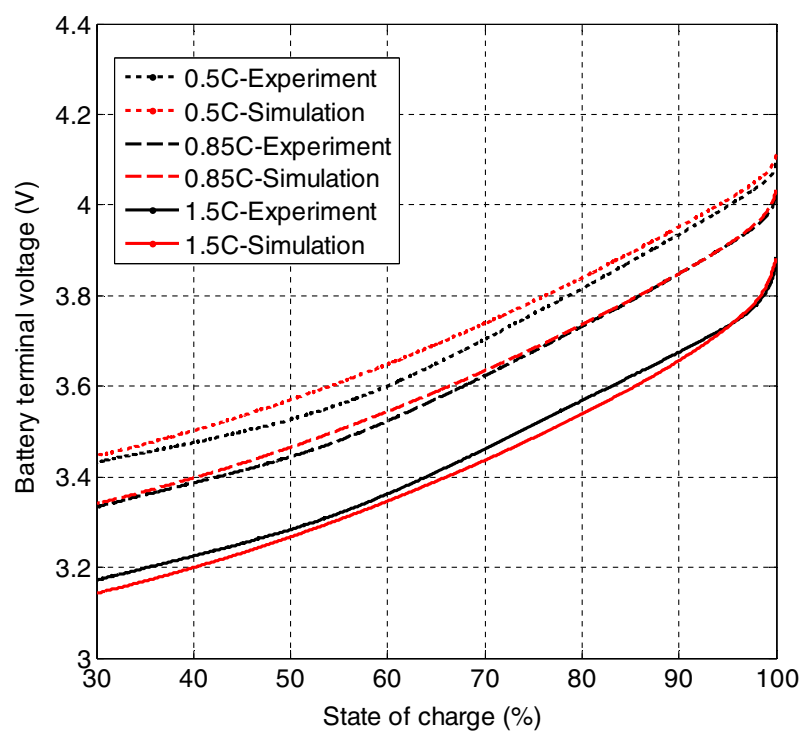

Figure 11. Validating simulation model under continuous discharge test.

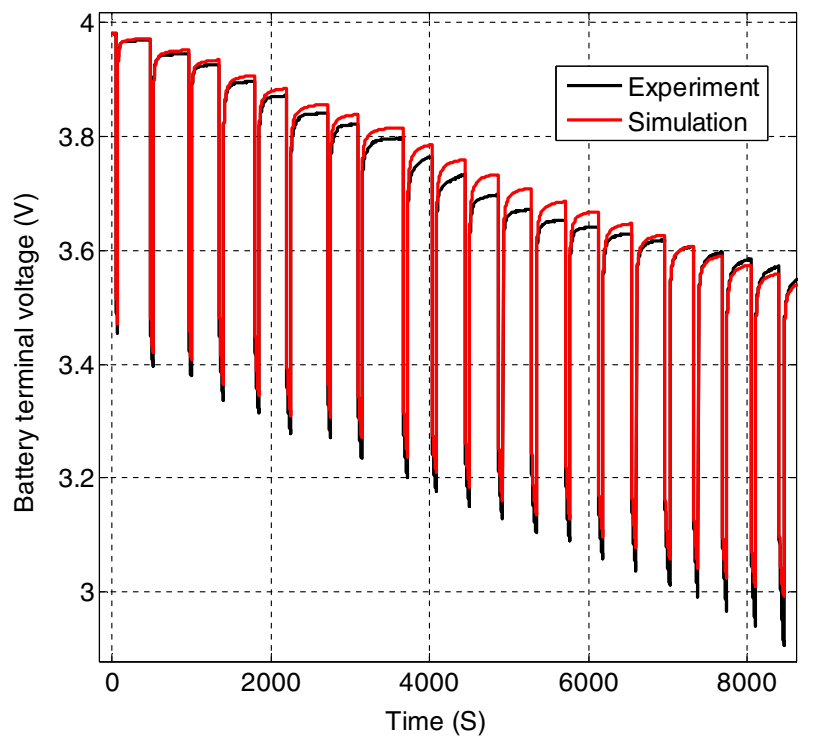

Figure 12. Validating simulation model under pulse discharge (2C) test.

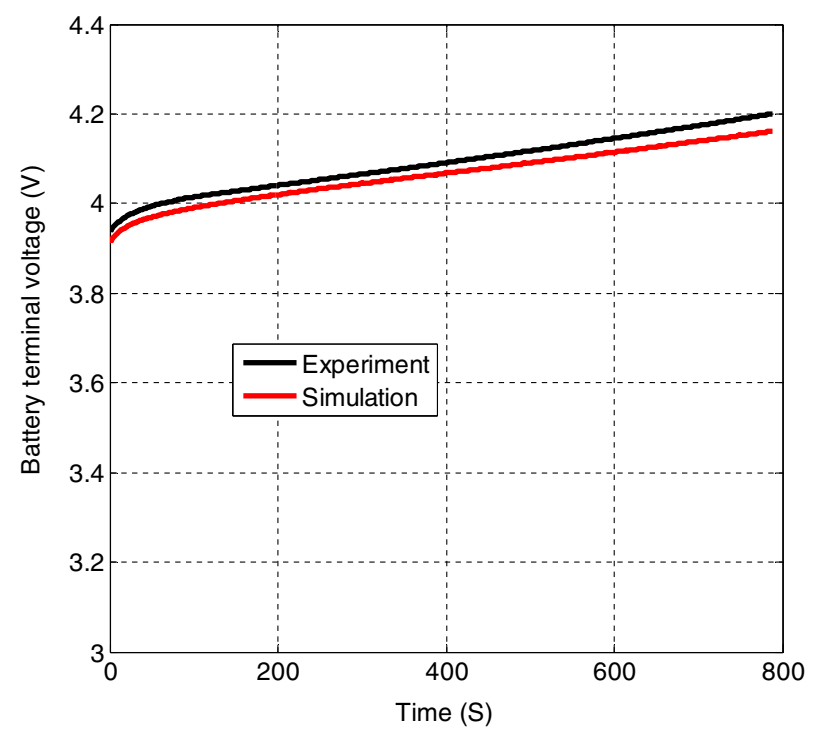

Figure 13. Validating simulation model under constant current charge (1C) test.

validated by matching the simulation results with experimental terminal voltage under discharge tests. figure 11 shows the results from simulation and experiment under different constant discharge currents. The terminal voltage matches reasonably well and the RMS values of error between experiment and simulation for $0.5 \mathrm{C}, 0.85 \mathrm{C}$ and $1.5 \mathrm{C}$ in the SOC range from $100 \%$ to $30 \%$ are $32 \mathrm{mV}$, $12.7 \mathrm{mV}$ and $22.6 \mathrm{mV}$ respectively. As the equation for CVS is obtained by averaging the experimental CVS values for various C-rates, the simulation and experimental results match reasonably well for all the $\mathrm{C}$-rates. The dynamic behavior of the simulation model is verified by comparing its results with the experimental pulse test, as shown in 
figure 12. The pulse test is conducted at $2 \mathrm{C}$-rate with discharge period of $60 \mathrm{~s}$ and relaxation period of $300 \mathrm{~s}$. It can be inferred from figure 12 that the proposed model is able to mimic the relaxation phenomenon closely with an RMS error of $21.6 \mathrm{mV}$ over duration of $8000 \mathrm{~s}$. For the pulse tests performed at $0.17 \mathrm{C}$ and $0.75 \mathrm{C}$ rates, the values of RMS error are found to be $32.3 \mathrm{mV}$ and $29 \mathrm{mV}$ respectively over the useful range of SOC. For constant current charging at $1 \mathrm{C}$ rate over duration of around $800 \mathrm{~s}$ till the upper cut-off voltage of $4.2 \mathrm{~V}$ the RMS error is $27 \mathrm{mV}$ (figure 13).

\section{Conclusion}

The proposed Li-ion battery model utilizes the features of both electrical circuit and analytical modeling techniques. Experiments are carried out on $3.7 \mathrm{~V}, 2200 \mathrm{mAh} \mathrm{Li-ion}$ cells. The model is based on measured BTV and impedance characteristics. The internal voltage CVS is realized as a second-order polynomial function of SOC to accurately depict the terminal characteristics as well as to maintain the simplicity of model. The impedance parameters are experimentally determined. Variation of internal parameters with SOC and C-rate is thoroughly investigated. To keep the model simple average values of impedance parameters are incorporated. The experimental and simulation data are compared to validate the accuracy of the model, under various discharge conditions. The model also mimics the dynamic characteristics of battery, with accuracy. As the model is accurate and simple, it can be employed in the real-time simulation of electric vehicles.

The series internal resistance $R_{\mathrm{S}}$ plays a key role in determining the battery performance. The value of $R_{\mathrm{S}}$ increases with the number of charge-discharge cycles. Therefore, it can be used as a parameter to evaluate the health of the battery. Estimating the state of health ( $\mathrm{SOH})$ of the battery is crucial to improve reliability of the vehicle and it is much simpler if it is based on the value of $R_{\mathrm{S}}$. The terminal characteristics of battery also depend on temperature. In this paper, the experiments are conducted at room temperature. The battery model is shown to match the measured battery characteristics for constant and pulse loading conditions. Future work will involve the battery $\mathrm{SOH}$ estimation by modeling the series resistance $R_{\mathrm{S}}$ as a function of battery aging. Incorporation of effect of temperature is also left for future work.

\section{Nomenclature}

$\begin{array}{ll}\text { SOC } & \text { State of charge } \\ \text { SOH } & \text { State of health } \\ \text { OCV } & \text { Open-circuit voltage } \\ \text { BTV } & \text { Battery terminal voltage }\end{array}$

\begin{tabular}{|c|c|}
\hline CVS & Controllable voltage source \\
\hline DOD & Depth of discharge \\
\hline$R_{\mathrm{S}}$ & Internal series resistance of the battery \\
\hline$R_{1}, C_{1}, R_{2}, C_{2}$ & $\begin{array}{l}\text { Transient response parameters of the } \\
\text { battery }\end{array}$ \\
\hline$R_{\mathrm{SD}}$ & Self-discharge resistor of the battery \\
\hline$R_{\mathrm{B}}$ & $\begin{array}{l}\text { Equivalent or base resistance of the } \\
\text { battery }\end{array}$ \\
\hline$C_{\mathrm{B}}$ & $\begin{array}{l}\text { Equivalent or base capacitance of the } \\
\text { battery }\end{array}$ \\
\hline$V_{\mathrm{N}}$ & Nominal voltage of the battery \\
\hline$V_{\mathrm{C}}$ & Cut-off voltage of the battery \\
\hline$Q_{\mathrm{N}}$ & Nominal capacity of the battery \\
\hline
\end{tabular}

\section{References}

[1] Nishi Y 2001 Lithium ion secondary batteries: Past 10 years and the future. J. Power Sources 100(1): 101-106

[2] Ritchie A G 2001 Recent developments and future prospects for lithium rechargeable batteries. J. Power Sources 96(1): 1-4

[3] Scrosati B and Garche J 2010 Lithium batteries: Status, prospects and future. J. Power Sources 195(9): 2419-2430

[4] Barsukov Y 2009 Secondary batteries-lithium rechargeable systems: Hazards and protection circuits. Encyclopedia of electrochemical power sources. Elsevier: 177-182

[5] Dubaniewicz T H and DuCarme J P 2013 Are lithium ion cells intrinsically safe? IEEE Trans. Ind. Appl. 49(6): 2451-2460

[6] Lisbona D and Snee T 2011 A review of hazards associated with primary lithium and lithium-ion batteries. Process Safety and Environmental Protection 89(6): 434-442

[7] Pistoia G 2010 Electric and hybrid vehicles: Power sources, models, sustainability, infrastructure, and the market, Great Britain, UK, Elsevier

[8] Barré A, Deguilhem B, Grolleau S, Gérard M, Suard F and Riu D 2013 A review on lithium-ion battery ageing mechanisms and estimations for automotive applications. J. Power Sources 241(1): 680-689

[9] Dubarry M, Liaw B Y, Chen M, Chyan S, Han K, Sie W and Wu S 2011 Identifying battery aging mechanisms in large format $\mathrm{Li}$ ion cells. J. Power Sources 196(7): 3420-3425

[10] Roscher M A, Assfalg J and Bohlen O S 2011 Detection of utilizable capacity deterioration in battery systems. IEEE Trans. Veh. Technol. 60(1): 98-103

[11] Vetter J, Winter M and Wohlfahrt-Mehrens M 2009 Secondary batteries - lithium rechargeable systems - lithiumion: Aging mechanisms. Encyclopaedia of electrochemical power sources, Elsevier. 393-403

[12] Wipke K B, Cuddy M R and Burch S D 1999 ADVISOR 2.1: A user-friendly advanced powertrain simulation using a combined backward/forward approach. IEEE Trans. Veh. Technol. 48(6): 1751-1761

[13] Doyle M, Fuller T F and Newman J 1993 Modeling of galvanostatic charge and discharge of the lithium/polymer/insertion cell. J. Electrochem. Soc. 140(6): 1526-1533 
[14] Doyle M and Newman J 1995 The use of mathematical modeling in the design of lithium/polymer battery systems. Electrochim. Acta 40(13-14): 2191-2196

[15] Fuller T F, Doyle M, and Newman J 1994 Simulation and optimization of the dual lithium-ion insertion cell. J. Electrochem. Soc. 141(1): 1-10

[16] Rao R, Vrudhula S and Rakhmatove D N 2003 Battery modeling for energy aware system design. Computer 36(12): 77-87

[17] Chiasserini C and Rao R R 2001 Energy efficient battery management. IEEE J. Sel. Areas Commun. 19(7): 1235-1245

[18] Rakhmatov D, Vrudhula S and Wallach D A 2003 A model for battery lifetime analysis for organizing applications on a pocket computer. IEEE Trans. VLSI Syst. 11(6): 1019-1030

[19] Rong P and Pedram M 2006 An analytical model for predicting the remaining battery capacity of lithium-ion batteries. IEEE Trans. VLSI Syst. 14(5): 441-451

[20] Buller S, Thele M, Doncker R W D and Karden E 2005 Impedance-based simulation models of supercapacitors and Li-ion batteries for power electronic applications. IEEE Trans. Industry Appl. 41(3): 742-747

[21] Rodrigues S, Munichandraiah N and Shukla A K 2000 A review of state-of-charge indication of batteries by means of ac impedance measurements. J. Power Sources 87(1): 12-20

[22] Benini L, Castelli G, Macci A, Macci E, Poncino M and Scarsi R 2001 Discrete-time battery models for system-level low-power design. IEEE Trans. VLSI Syst. 9(5): 630-640
[23] Gold S 1997 A pspice macromodel for lithium-ion batteries. In: Twelfth Annual Battery Conference on Applications and Advances. 215-222

[24] Chen S X, Gooi H B, Xia N and Wang M Q 2012 Modelling of lithium-ion battery for online energy management systems. IET Electr. Syst. Transp. 2(4): 202-210

[25] Coleman M, Lee C K, Zhu C and Hurley W G 2007 State-ofcharge determination from EMF voltage estimation: Using impedance, terminal voltage, and current for lead-acid and lithium-ion batteries. IEEE Trans. Ind. Electron. 54(5): 2550-2557

[26] Einhorn M, Conte F V, Kral C and Fleig J 2013 Comparison, selection, and parameterization of electrical battery models for automotive applications. IEEE Trans. Power Electron 28(3): 1429-1437

[27] Raman S R, Saritha B and John V 2013 Computationally efficient and accurate modeling of Li-ion battery. IEEE ISGT, Bangalore, India. 1-6

[28] Chen M and Rincon-Mora G A 2006 Accurate electrical battery model capable of predicting runtime and I-V performance. IEEE Trans. Energy Convers. 21(2): 504-511

[29] Zhang H and Chow M Y 2010 Comprehensive dynamic battery modeling for PHEV applications. IEEE PES General Meeting. 1-6

[30] Wolberg J 2006 Data analysis using the method of least squares: Extracting the most information from experiments, Springer 